\title{
Staphylococcus lugdunensis BACTEREMIA IN HEMODIALYSIS PATIENTS
}

\section{MALLAPPALLIL M.C. ${ }^{1}$, MORO SALIFU1, YALEMZEWD WOREDEKAL ${ }^{1}$, MARTIN KRAMER ${ }^{1}$, PHILLIPS A.R. ${ }^{1}$, SAURABH MALHORTA, KHALED ABU-LAWI2 AND BLUTH M.H. ${ }^{3}$}

\author{
1 Department of Medicine - Division of Nephrology and Department of Microbiology, Kings County Hospital Center and SUNY Downstate \\ Medical Center, New York, USA. \\ 2Department of Pathology, Kings County Hospital Center, Brooklyn, New York, USA. \\ ${ }^{3}$ Department of Pathology, Wayne State University School of Medicine, Detroit, MI 48201. \\ *Corresponding Author: Email- mbluth@med.wayne.edu
}

Received: February 10, 2012; Accepted: March 15, 2012

\begin{abstract}
Staphylococcus lugdunensis can cause virulent infections in immunosuppressed individuals. Here we describe a retrospective analysis of hemodialysis patients with Staphylococcus lugdunensis bacteremia admitted to a New York City hospital from January 2005 to December 2010. Six cases of S. lugdunensis bacteremia were identified. The majority had received antibiotic therapy for at least several weeks. None of them developed endocarditis or other serious complications. Four had their tunneled permanent dialysis catheters changed or removed. This pathogen is rare, but could often fail medical therapy alone and may require surgical intervention. This can be avoided with surveillance and early intervention in patients with high risk for infection.
\end{abstract}

Key words- Staphylococcus lugdunensis, Staphylococcus, bacteremia, infection, hemodialysis.

Citation: Mallappallil M.C., et al. (2012) Staphylococcus lugdunensis bacteremia in hemodialysis patients. International Journal of Microbiology Research, ISSN: 0975-5276 \& E-ISSN:0975-9174, Volume 4, Issue 2, pp.-178-181.

Copyright: Copyright@2012 Mallappallil M.C., et al. This is an open-access article distributed under the terms of the Creative Commons Attribution License, which permits unrestricted use, distribution, and reproduction in any medium, provided the original author and source are credited.

\section{Introduction}

S. Iugdunensis is coagulase negative staphylococcus described by Fleurette et al in 1989 [1] but has been recently described as an emerging human pathogen [2]. While characterized as coagulase negative, because it does not produce coagulase, there is a membrane bound form of the enzyme in some isolates that can cause it to be misidentified as $\mathrm{S}$. aureus [3].

Although it is considered a skin normal flora, it can cause virulent infections usually in immunosuppressed patients with implanted medical devices including bone replacements and cardiac valve replacements [4]. We report cases of $S$. lugdunensis bacteremia in hemodialysis patients with permanent tunneled cuffed catheters admitted to a New York City hospital.

\section{Methods}

At least one set of blood cultures were taken from each patient. Blood culture was collected in an adult Bac T/ALERT blood culture set, and upon receipt by the Microbiology laboratory, it was incubated in Bac T/ALERT instrument. Positive blood cultures were then Gram stained and sub cultured on TSA, MacConkey and Chocolate plated media. Next Day Positive cultures on the plate were identified using Walk Away MicroScan panels.
Results
Patients on hemodialysis who were infected with S. lugdunensis as a cause of bacteremia were identified. As shown in Table 1, all patients were African American and represented both genders equally. The average patient age was 53.8 years and the majority $(83 \%)$ had hypertension among other co-morbidities. Half of the patients had their catheter placement at the NYC hospitals or its affiliates and $75 \%$ of patients had their hemodialysis treatment site elsewhere. The majority of patients were on dialysis for 2 years or less and none of the patients suffered any complications at time of discharge. Antibiotic sensitivities of S. lugdunensis cul- tured from infected patients are shown in Table 2. Isolates ob- tained from all patients were sensitive to ceftriaxone, clindamycin, oxacillin, rifampin, trimethoprim/sulfamethoxisole and vancomycin and resistant to penicillin. Of those tested, one of six patients was separately resistant to gentamycin and tetracycline. A brief sum- mary of each of the patients infected with S. lugdunensis follows: 
Table 1- Patient characteristics

\begin{tabular}{|c|c|c|c|c|c|c|}
\hline Case number & 1 & 2 & 3 & 4 & 5 & 6 \\
\hline Age(yrs) & 63 & 47 & 56 & 64 & 61 & 32 \\
\hline Race/Ethnicity & AA & AA & AA & $\mathrm{AA}$ & $\mathrm{AA}$ & $\mathrm{AA}$ \\
\hline Gender & Female & Female & Male & Female & Male & Male \\
\hline Started HD & 2001 & 2006 & 2005 & 2006 & $?$ & $\begin{array}{l}1997 \\
2009\end{array}$ \\
\hline Co morbidities & HTN,CVA & HIV & $\begin{array}{l}\text { HTN, } \\
\text { CVA }\end{array}$ & $\begin{array}{l}\text { HTN, } \\
\text { DM }\end{array}$ & HTN & $\begin{array}{l}\text { HTN, } \\
\text { DM }\end{array}$ \\
\hline $\begin{array}{l}\text { Date of Cathe- } \\
\text { ter placement }\end{array}$ & 2007 & 2006 & 2006 & 2006 & $?$ & 2009 \\
\hline Site of HD & Our hosp & $\begin{array}{l}\text { Outside } \\
\text { hosp }\end{array}$ & $\begin{array}{l}\text { Our } \\
\text { hosp }\end{array}$ & $\begin{array}{l}\text { Outside } \\
\text { hosp }\end{array}$ & $\begin{array}{l}\text { Outside } \\
\text { hosp }\end{array}$ & $\begin{array}{l}\text { Outside } \\
\text { hosp }\end{array}$ \\
\hline $\begin{array}{l}\text { Site of Catheter } \\
\text { placement }\end{array}$ & Our hosp & $\begin{array}{l}\text { Our } \\
\text { hosp } \\
\text { (LIJ) }\end{array}$ & $\begin{array}{l}\text { Our } \\
\text { hosp }\end{array}$ & $\begin{array}{l}\text { Outside } \\
\text { hosp }\end{array}$ & $\begin{array}{l}\text { Outside } \\
\text { hosp }\end{array}$ & $\begin{array}{l}\text { Outside } \\
\text { hosp }\end{array}$ \\
\hline $\begin{array}{l}\text { Years on } \\
\text { dialysis }\end{array}$ & 6 & 1 & 1 & 2 & $?$ & 0.5 \\
\hline $\begin{array}{l}\text { History of } \\
\text { previous } \\
\text { bacteremia }\end{array}$ & $?$ & Y & $Y$ & ? & ? & Y \\
\hline Treatment & $?$ & $?$ & $?$ & oxacillin & $?$ & ? \\
\hline
\end{tabular}

Key: $A A=$ African American, HTN= hypertension, $Y=y e s, N=n o, ?=$ unknown, hosp=hospital

Table 2- Sensitivities of S. Lugdunensis

\begin{tabular}{llllllll} 
Case & 1 & $\mathbf{2}$ & $\mathbf{3}$ & $\mathbf{4}$ & $\mathbf{5}$ & $\mathbf{6}$ \\
Ceftriaxone & $\mathrm{S}$ & $\mathrm{S}$ & $\mathrm{S}$ & $\mathrm{S}$ & $\mathrm{S}$ & $\mathrm{S}$ \\
Clindamycin & $\mathrm{S}$ & $\mathrm{S}$ & $\mathrm{S}$ & $\mathrm{S}$ & $\mathrm{S}$ & $\mathrm{S}$ \\
Gatifloxacin & $\mathrm{S}$ & - & - & - & - & - \\
Erythromycin & - & - & $\mathrm{S}$ & - & $\mathrm{S}$ & $\mathrm{S}$ \\
Ciprofloxaxin & - & - & - & - & - & - \\
Gentamicin & $\mathrm{S}$ & $\mathrm{S}$ & $\mathrm{S}$ & $\mathrm{R}$ & $\mathrm{S}$ & $\mathrm{S}$ \\
Levofloxacin & $\mathrm{S}$ & $\mathrm{S}$ & $\mathrm{S}$ & $\mathrm{S}$ & - & - \\
Moxifloxacin & - & - & - & - & $\mathrm{S}$ & $\mathrm{S}$ \\
Oxacillin & $\mathrm{S}$ & $\mathrm{S}$ & $\mathrm{S}$ & $\mathrm{S}$ & $\mathrm{S}$ & $\mathrm{S}$ \\
Penicillin & $\mathrm{R}$ & $\mathrm{R}$ & $\mathrm{R}$ & $\mathrm{R}$ & $\mathrm{R}$ & $\mathrm{R}$ \\
Rifampin & $\mathrm{S}$ & $\mathrm{S}$ & $\mathrm{S}$ & $\mathrm{S}$ & $\mathrm{S}$ & $\mathrm{S}$ \\
Tetracycline & $\mathrm{S}$ & $\mathrm{S}$ & $\mathrm{R}$ & $\mathrm{S}$ & $\mathrm{S}$ & $\mathrm{S}$ \\
Trimeth/sulfa & $\mathrm{S}$ & $\mathrm{S}$ & $\mathrm{S}$ & $\mathrm{S}$ & $\mathrm{S}$ & $\mathrm{S}$ \\
Vancomycin & $\mathrm{S}$ & $\mathrm{S}$ & $\mathrm{S}$ & $\mathrm{S}$ & $\mathrm{S}$ & $\mathrm{S}$ \\
\hline
\end{tabular}

Key: $S=$ sensitive, $R=$ resistant, - = not tested

\section{Clinical Cases}

The first case was a 63 year old woman with a history of hypertension, end stage renal disease (ESRD) on hemodialysis (HD) for six years via a tunneled dialysis catheter (TDC) presented to the ER with bleeding from her dialysis catheter site, fever and chills for two days. Following balloon dilation because of superior vena cava (SVC) stenosis, the patient had a new right internal jugular TDC placed. She was on coumadin since 2006 for the SVC stenosis. Her other medical history was significant for a previous cerebrovascular accident and pancytopenia which was being worked up by the hematology/oncology service. Vital signs were significant for a blood pressure of $165 / 85 \mathrm{mmHg}$. The site over her right hemodialysis catheter was warm, non-tender with no pus and a hematoma was present. She had a systolic flow murmur (previously identified) on cardiac auscultation. Blood cultures were drawn and she was given vancomycin 1gram and gentamicin 240 mg empirically. Her WBC count was 3800/uL with $11 \%$ neutrophils, but no bands. Blood cultures grew S. lugdunensis resistant to penicillin. The hemodialysis catheter was exchanged for a new one. She also had angioplasty with stent placement in the superior vena cava. Follow up blood cultures were negative. Transthoracic echocardiography (TTE) was negative for endocarditis.

The second case was a 47 year old woman on highly active antiretroviral therapy for HIV (CD4 count of 467) and with ESRD on hemodialysis for 2 years. She developed palpitations during her dialysis session and was sent to the ER. She was febrile and had a maturing arterio-venous fistula. Her blood pressure was $145 / 59 \mathrm{mmHg}$, heart rate 80 and WBC 6000/uL with 59\% neutrophils without bands. She was given empiric vancomycin and gentamicin. Blood cultures grew S. lugdunensis resistant to penicillin. Cultures cleared with antibiotics. The hemodialysis catheter was not immediately removed. Once the patient's fistula was matured and utilized, the catheter was removed. Repeat blood cultures were negative.

The third case was a 55 year old man with ESRD on HD for 1 year, hypertension and an old cerebrovascular accident was admitted after a brief syncopal episode. He was severely anemic (hemoglobin of $3.5 \mathrm{~g} / \mathrm{dL}$ ) and uremic. He had been on a six week course of antibiotic therapy for a dialysis catheter-related Staphylococcus aureus bacteremia. His TDC was removed the month prior and he was being dialyzed via temporary femoral access until he had negative blood cultures. His vital signs were unremarkable and he was adequately transfused and dialyzed. One blood culture on the current admission was positive for $S$. lugdunensis. TTE in the month prior was negative for endocarditis. Subsequent blood cultures were negative and a new permanent dialysis catheter was placed in late June. The patient eventually received an arterio-venous fistula.

The fourth case was a 67 year old woman with a history of hypertension (HTN), type 2 diabetes mellitus (DM) and ESRD on HD via TDC for 2 years at a non-KCH center who presented to our emergency room with altered mental status. She had missed 4 dialysis sessions. She had no evidence of fluid overload, pericardial rub or asterixis. She was afebrile and her TDC site was clean. Lab work revealed hyperkalemia $\left(\mathrm{K}^{+}, 6.3 \mathrm{mEq} / \mathrm{L}\right)$ without ECG changes, BUN $184 \mathrm{mg} / \mathrm{dL}$, creatinine $19.06 \mathrm{mg} / \mathrm{dL}$ and bicarbonate $16 \mathrm{mg} / \mathrm{dL}$ but normal leuckocyte count without bands. Head CT showed an area of focal hypodensity of indeterminate age. She was emergently dialysed that day and for a few days afterwards with mild improvement in her neurological status. During her delirium, she removed her TDC. Blood cultures grew S. lugdunensis. TTE did not show any vegetations. Transesophageal echocardiogram (TEE) was planned but not performed since the patient's hospitalization was complicated by two ICU admissions for hypotension, gastrointestinal bleeding ( 3 duodenal ulcers) and sepsis (left gluteal abscess from Citrobacter). Subsequent blood cultures were negative after treatment with oxacillin for 4 weeks. She was discharged to a nursing home.

The fifth case was a 61 year old man with a history of hypertension and ESRD on HD via a TDC who presented to our ER with complaints of subjective fevers, right shoulder and back pain after dialysis. He was febrile and physical exam was unremarkable. Vancomycin $1 \mathrm{~g}$ and gentamicin 90mg were given empirically. Blood cultures were initially negative and patient's fever resolved. No vegetations were seen on TTE. The patient was stable throughout his 24 hour hospital stay and was subsequently discharged. The following day final microbiological report from the blood cultures showed $S$. lugdunensis. The patient was recalled and repeat cultures done. These were negative. No TEE was per- 
formed and the catheter was not removed.

The sixth case was a 32 year old man with HTN, DM, and ESRD since 1997, who presented to the ER complaining of malaise, intermittent subjective fevers and chills for 1 month during or after dialysis. He recently restarted dialysis for 3 months after a failed deceased donor renal transplant (DDRT) after 6 years. A femoral tunneled catheter was inserted because he had multiple failed attempts at upper extremities permanent access. He'd missed a few dialysis sessions over the last month because he wanted to avoid the above symptoms. Physical exam was significant for hypertension $(200 / 109 \mathrm{mmHg})$ but he was afebrile with a clean femoral dialysis catheter site and no evidence of uremia. Labs revealed potassium of $6.8 \mathrm{mEq} / \mathrm{L}$ and creatinine of $18.56 \mathrm{mg} / \mathrm{dL}$ but a normal leukocyte count and no bands. He was dialysed emergently.

The rest of his hospitalization was uneventful and at the time of discharge all blood cultures were negative. The team made a decision not to administer antibiotics. He was to follow up with his vascular surgeon the following week with recommendations from the team to have the TDC changed. No TTE or TEE was performed. Final blood culture reports were significant for S. Lugdenensis and Acinetobacter baumannii from 1 blood culture (unclear if peripheral vs. TDC site). It is not known if the patient had the TDC removed and a new access placed.

\section{Discussion}

Catheter- related blood stream infections (CRBSI) occur in hemodialysis patients with central access catheters. The incidence of dialysis-related CRBSI is reported to be 2.5-5.5 cases per 1,000 catheter days, or 0.9-2.0 episodes per patient-year [5]. CRBSI increase hospital cost and length of stay, thus highlighting the importance of prevention and awareness. Hemodialysis patients may benefit from an arterio-venous fistula or graft for permanent access instead of a central catheter but this is not always possible for those with poor native veins or significant venous stenosis $[5,6]$.

Catheter contamination can occur through one of 4 routes: i) migration of skin organisms at the insertion site, ii) direct contamination of the catheter or catheter hub - common for tunneled catheters, iii) hematogenous seeding from another infection focus, and iv) infusion of contaminated infusate [7]. The majority of these infections are due to $S$. aureus and $S$. epidermidis.

$S$. lugdunensis is one of the organisms found as part of the normal human skin flora. Despite being coagulase negative, it causes infections more akin to $S$. aureus than other coagulase negative staphylococci (i.e. S epidermidis). This virulence is attributable to the production of several virulence factors, including delta toxinlike hemolytic peptide, adhesions and enzymes among others [8]. Infections seen with this organism can involve skin and soft tissue, in addition to the eyes, urinary tract, central nervous system, bone and joint, perineum and heart valves [2]. Bacteremia with this organism has been noted to cause endocarditis (mostly left-sided) in up to $50 \%$ of patients with native valves leading to rapid tissue destruction, high mortality and resulting in removal of the infected valves [9]. S. Lugdunensis bacteremia has also been reported in hemodialysis patients in the setting of pulmonary valve endocarditis [10].

In our infection control surveillance in 2005, we had no reports of
S. lugdunensis bacteremia occurring in dialysis patients admitted to the hospital. Between January 2006 and December 2010 twenty seven cases of this bacteremia were noted, six were among hemodialysis patients all with tunneled dialysis catheters. Of these $50 \%$ were male and half of the patients had a previous history of bacteremia. Four had their tunneled catheters removed. No information was available for the remaining two.

All our cases were admitted acutely and the majority were from outside dialysis centers. Three had multiple failed accesses and two were awaiting placement or maturation of an arterio-venous fistula. The majority of patients also had varying levels of immunosuppression apart from having ESRD: two were diabetic; one had pancytopenia and the other had HIV. To our knowledge patient survival at the time of discharge was $100 \%$.

In the past, as observed in our institution, few cases of infection were noted with this organism. Previous reports by Schoheyder and colleagues [11] revealed that in a retrospective study performed on 978 coagulase negative Staphylococcal blood culture isolates, that none of the 978 cases were identified as S. Iugdunensis . However, data generated over ten years later by Anguera et al., reported ten cases of $S$. lugdunensis in 912 consecutive patients with infective endocarditis over a 13 year period between 1990 and 2003 [12]. Further, resistance patterns for this organism have changed since the earlier observations by Herscheline et al [13] which reported 59 clinical isolates; where all strains were sensitive to penicillin, oxacillin and gentamicin. In contrast, all of our isolates were resistant to penicillin and only two separate patient's isolates were resistant to gentamicin and tetracycline, respectively.

Many of the patients had a known history of bacteremia and/or diabetes. Consistent with our findings, Katneni et al [5] suggest that risk factors for CRBSI in hemodialysis patients include previous bacteremia and diabetes mellitus. Other risk factors include long duration of catheter use, $S$. aureus nasal colonization, older age, higher total intravenous iron dose, lower hemoglobin and serum albumin levels and recent hospitalization [5].

There are several limitations identified in the current study. First we did not have nasal swabs or cultures from other sources to exclude hematogenous sources of $S$. lugdunensis. Second we did not have information on patient catheter care which would point to increased risk for catheter hub contamination. Third, it is not clear for most of the patients why a more permanent dialysis access such as an arterio-venous fistula or graft was not present, thereby reducing the likelihood for CRBSI. Nonetheless, the recent increases in the incidence of $S$. lugdunensis bacteremia are important to recognize. It stresses the importance of vigilant dialysis catheter care through patient and health care professional education in conjunction with other measures to prevent catheter associated blood stream infections [7]. Further, ideally we should aim to have an arterio-venous fistula or graft placement in all chronic hemodialysis patients where applicable to reduce the risk of $S$. lugdunensis infection and bacteremia in general.

\section{Conclusion}

In conclusion, we highlight the characterization of S.lugdunensis bacteremia in hemodialysis patients. The virulent nature of this emerging clinical pathogen calls for increased awareness strict surveillance and early action. 


\section{References}

[1] Fleurette J., Bès M., Brun Y., Freney J., Forey F., Coulet M., Reverdy M.E., Etienne J. (1989) Res Microbiol. 140(2),107118.

[2] Klotchko A., Wallace M.R., Licitra C., Sieger B. (2011) South Med J. 104(7),509-514.

[3] Frank K.L., Del Pozo J.L., Patel R. (2008) Clin Microbiol Rev. 21(1), 111-133.

[4] Seifert H., Oltmanns D., Becker K., Wisplinghoff H., von Eiff C. (2005) Emerg Infect Dis. 11(8),1283-1286.

[5] Katneni R., Hedayati S.S. (2007) Nat Clin Pract Nephrol. 3(5), 256-266.

[6] National Kidney Foundation. III. (2000) Am. J. Kidney Dis. 37, S137-S181.

[7] O'Grady N.P., Alexander M., Burns L.A., Dellinger E.P., Garland J., Heard S.O., Lipsett P.A., Masur H., Mermel L.A., Pearson M.L., Raad I.I., Randolph A.G., Rupp M.E., Saint S. (2011) Infect. Dis., 52(9), e162-e193.

[8] Szabados F., Nowotny Y., Marlinghaus L., Korte M., Neumann S., Kaase M., Gatermann S.G. (2011) BMC Res Notes. 8(4), 113.

[9] Zinkernagel A.S., Zinkernagel M.S., Elzi M.V., Genoni M., Gubler J., Zbinden R., Mueller N.J. (2008) Infection 36(4), 314 $-321$.

[10]Kamaraju S., Nelson K., Williams D.N., Ayenew W., Modi K.S. (1999) Am. J. Nephro 19(5), 605-608.

[11]Schønheyder H.C., Hansen V.K., Asschenfeldt P., Rosdahl V.T. (1993) APMIS 101(10), 802-804.

[12]Anguera I., Del Río A., Miró J.M., Matínez-Lacasa X., Marco F., Gumá J.R., Quaglio G., Claramonte X., Moreno A., Mestres C.A., Mauri E., Azqueta M., Benito N., García-de la María C., Almela M., Jiménez-Expósito M.J., Sued O., De Lazzari E., Gatell J.M. (2005) Heart 91(2), e10.

[13]Herchline T.E., Barnishan J., Ayers L.W., Fass R.J. (1990) Penicillinase production and in vitro susceptibilities of Staphylococcus lugdunensis Antimicrob Agents Chemother, 34(12), 2434-2435. 